\title{
Effects of Pitavastatin, a 3-Hydroxy-3-Methylglutaryl Coenzyme A Reductase Inhibitor, on Cardio-Ankle Vascular Index in Type 2 Diabetic Patients
}

\author{
Yoh Miyashita, Kei Endo, Atsuhito Saiki, Noriko Ban, Takashi Yamaguchi, Hidetoshi Kawana, \\ Daiji Nagayama, Masahiro Ohira, Tomokazu Oyama, and Kohji Shirai
}

Departments of Internal Medicine, Sakura Medical Center, School of Medicine, Toho University, Chiba, Japan

\begin{abstract}
Aim: A novel device has been developed for measuring the cardio-ankle vascular index (CAVI) as an indicator of arterial stiffness. In this study, we evaluated the effect of pitavastatin on CAVI in type 2 diabetic patients.

Methods: Forty-five type 2 diabetes mellitus patients with low-density lipoprotein cholesterolemia were enrolled and treated with pitavastatin $2 \mathrm{mg} /$ day for 12 months. Before and after pitavastatin administration, HbA1c, serum lipids, serum malondialdehyde-LDL (MDA-LDL), urinary 8-hydroxy2'-deoxyguanosine (8-OHdG) and CAVI were measured.

Results: After pitavastatin treatment for 12 months, significant decreases in 8-OHdG, MDA-LDL and CAVI were observed. $\triangle$ CAVI significantly correlated with $\triangle$ MDA-LDL.

Conclusions: In type 2 diabetic patients, pitavastatin may have an oxidative stress-reducing effect, especially in a state of enhanced oxidative stress, and CAVI may be useful as a routine test for the diagnosis and therapeutic monitoring of atherosclerosis.
\end{abstract}

J Atheroscler Thromb, 2009; 16:539-545.

Key words; Pitavastatin, Oxidative stress, 8-hydroxy-2'-deoxyguanosine, Malondialdehyde-LDL, Cardio-ankle vascular index

\section{Introduction}

Diabetic patients complicated with high low-density lipoprotein cholesterol (LDL-C) are at increased risk of macroangiopathy ${ }^{1,2)}$; therefore, in diabetic patients, intensive LDL-C-lowering therapy is needed to prevent the progression of atherosclerosis. 3-Hydroxy3-methylglutaryl coenzyme A reductase inhibitors (statins) are known to be effective in lowering LDL-C. Among the statins, pitavastatin has a potent LDL-Clowering effect. Various studies have reported significant decreases in the frequency of coronary heart disease by statin therapy ${ }^{3-5}$. Thus, the main purpose of

Address for correspondence: Yoh Miyashita, Department of Internal Medicine, Sakura Medical Center, School of Medicine, Toho University, 564-1 Shimoshizu, Sakura-City, Chiba, 2850841, Japan

E-mail: mumon@sf6.so-net.ne.jp

Received: August 20, 2008

Accepted for publication: March 5, 2009
LDL-C-lowering therapy is to reduce the risks of onset and aggravation of atherosclerotic diseases, and evaluation of vascular function is needed to monitor the therapeutic effect.

Pulse wave velocity (PWV) and the stiffness parameter $\beta$ evaluated by the change in diameter of the vessel wall are used to evaluate atherosclerosis or vascular function ${ }^{6-9)}$. The problem with PWV in clinical use is that PWV itself essentially depends on blood pressure. Although Hasegawa et al. ${ }^{8)}$ established the aortic PWV method, which is independent of blood pressure, this method has several drawbacks, such as difficulty in finding the notch of the pulse wave, need for technical skill, and low reproducibility. The stiffness parameter $\beta$ is based on the change in vascular diameter corresponding to arterial pressure variance ${ }^{7,10)}$, and the value does not depend on blood pressure ${ }^{11)}$; however, there are also various problems such as $\beta$ reflecting the local property of a segment of the artery and the need for special ultrasonic equipment. 
Recently, a novel arterial stiffness parameter, termed cardio-ankle vascular index (CAVI), has been developed, which essentially reflects the stiffness of the aorta, femoral artery and tibial artery ${ }^{12)}$. CAVI is independent of blood pressure, and has adequate reproducibility for clinical use ${ }^{12}$. Furthermore, no special technique is required to measure CAVI. Several reports have demonstrated the usefulness of CAVI to detect atherosclerotic diseases ${ }^{12-15)}$.

In the present study, we evaluated the effect of pitavastatin on CAVI in type 2 diabetic patients with high LDL-C.

\section{Subjects and Methods}

\section{Subjects}

A randomized, open study was performed. Fortyfive type 2 diabetes mellitus patients with high LDL-C, who attended Sakura Medical Center of Toho University as outpatients, were enrolled. Patients were excluded if they had received insulin therapy, or had diabetic retinopathy, nephropathy or previous cardiovascular and cerebrovascular diseases. The enrolled subjects were treated with pitavastatin $2 \mathrm{mg}$ /day for 12 months. The clinical profile of the subjects is shown in Table 1. During the study period, all patients maintained the same diet and exercise therapies, and did not change medications. All subjects received nutrition education from a dietitian every month. This study was approved by the institutional review board. The purpose of this study was explained to the subjects, and consent was obtained for participation in the study and also for release of the study data.

\section{Measurement of Body Weight and Blood Pressure}

Body weight and blood pressure were measured in the morning after 12 hours of fasting. Blood pressure was measured at least twice in a sitting position.

\section{Assay of HbAlc and Serum Lipids}

Blood samples were collected in the morning after 12 hours of fasting. Serum was separated within 1 hour, and samples were used to measure the following chemical parameters. Stable and unstable fractions of glycosylated hemoglobin (HbA1c) were measured by high pressure liquid chromatography using the HiAuto A1c kit (Kyoto Daiichi Kagaku, Kyoto, Japan). Data of the stable type were used in the present analysis. Total cholesterol (TC), triglyceride (TG) and LDL-C were measured with an automatic analyzer (Hitachi 7150; Hitachi Tokyo, Japan). High-density lipoprotein cholesterol (HDL-C) was measured by the selective inhibition method (Daiichi Pure Chemicals,
Table 1. Clinical parameters of subjects before and after pitavastatin treatment

\begin{tabular}{lcc}
\hline & Before & After \\
\hline $\mathrm{n}$ (male/female) & $45(19 / 26)$ & \\
Age & $65.5 \pm 6.2$ & \\
Body weight $(\mathrm{kg})$ & $55.8 \pm 6.9$ & $55.3 \pm 7.7$ \\
BMI $\left(\mathrm{kg} / \mathrm{m}^{2}\right)$ & $22.5 \pm 1.8$ & $22.3 \pm 2.6$ \\
Blood pressure (mmHg) & & \\
$\quad$ systolic & $125 \pm 12$ & $126 \pm 13$ \\
$\quad$ diastolic & $78 \pm 7$ & $75 \pm 7$ \\
Hemoglobin A1c (\%) & $6.9 \pm 1.4$ & $6.7 \pm 1.2$ \\
Total cholesterol (mg/dL) & $246 \pm 24$ & $203 \pm 31^{* *}$ \\
Triglyceride (mg/dL) & $146 \pm 54$ & $140 \pm 53$ \\
HDL-cholesterol (mg/dL) & $50.8 \pm 11.6$ & $56.2 \pm 12.4^{*}$ \\
LDL-cholesterol (mg/dL) & $166 \pm 22$ & $127 \pm 28^{* *}$ \\
Antihypertensives ${ }^{\mathrm{a}}$ [no. of subjects (\%)] & $16(36 \%)$ & \\
Anti-diabetic agents [no. of subjects (\%)] & & \\
$\quad$ Sulfonylureas & $32(71 \%)$ & \\
Thiazolidinedione & $4(9 \%)$ & \\
$\quad$ Alpha-glucosidase inhibitor & $10(22 \%)$ & \\
$\quad$ Biguanide & $16(36 \%)$ & \\
Smoking [no. of subjects (\%)] & $3(7 \%)$ & \\
\hline
\end{tabular}

Data: mean $\pm S D$;

a all angiotensin II receptor blockers; ${ }^{*} p<0.05,{ }^{* *} p<0.01$ by paired $t$-test

Tokyo) ${ }^{16)}$.

Assay of Serum Malondialdehyde-LDL

Serum malondialdehyde-LDL (MDA-LDL) was assayed by ELISA as described previously ${ }^{17}$. Monoclonal antibodies against MDA-LDL (ML25) and apo B (AB16) were used. These antibodies and 2-15\% nondenaturing polyacrylamide gels were obtained from Daiichi Chemicals Co. (Tokyo, Japan).

\section{Urinary 8-OHdG Analysis}

Urine samples were centrifuged at $800 \mathrm{~g}$ for 10 min and the supernatant was used to determine 8-hydroxy-2'-deoxyguanosine (8-OHdG) by a competitive enzyme-linked immunosorbent assay (8-Hydroxydeoxyguanosine Check; Japan Institute for the Control of Aging, Shizuoka, Japan). The monoclonal antibody has been characterized and found to be specific for $8-\mathrm{OHdG}^{18)}$. The results were adjusted for creatinine (per $\mathrm{mg} \mathrm{Cr}$ ) measured in the same urine sample.

\section{Measurement of CAVI}

CAVI was measured using a VaSera CAVI instrument (Fukuda Denshi Co. Ltd., Tokyo) by the meth- 
A

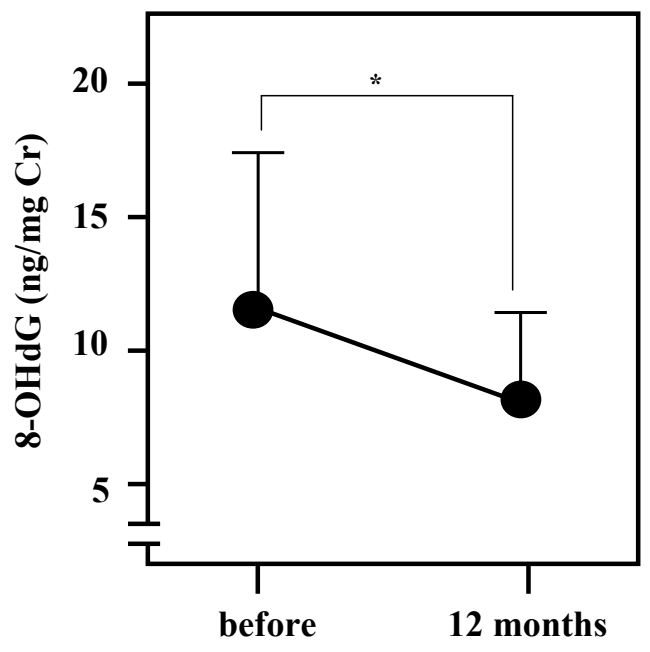

Pitavastatin administration

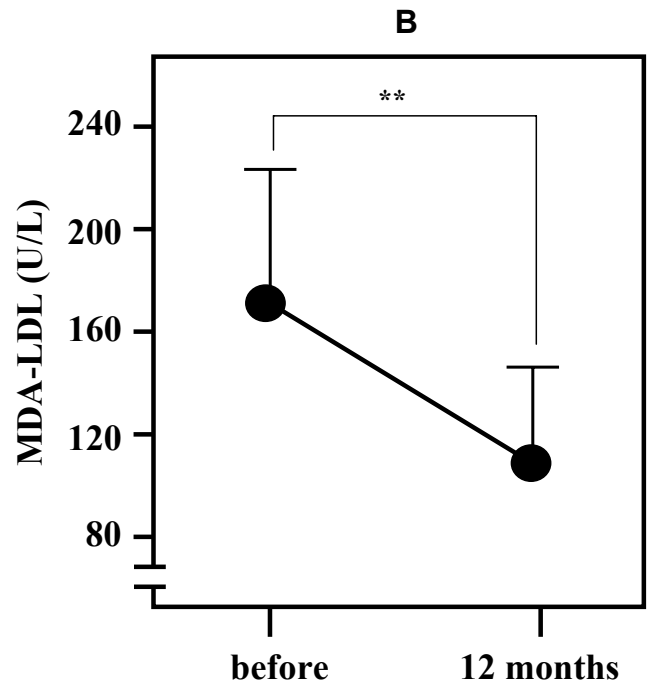

Pitavastatin administration

Fig. 1. Changes in urinary $8-\mathrm{OHdG}(\mathrm{A})$ and serum MDA-LDL (B) after pitavastatin treatment for 12 months.

Data are presented as the mean \pm S.D. ${ }^{*} p<0.05 ;{ }^{* *} p<0.01$ vs before treatment, paired $t$-test.

ods described previously ${ }^{12)}$. CAVI was measured in the morning after 12 hours of fasting. Briefly, cuffs were applied to bilateral upper arms and ankles, with the subject supine and the head held in the midline position. Examinations were performed after resting for 10 minutes. To detect brachial and ankle pulse waves with cuffs, a low cuff pressure of 30 to $50 \mathrm{mmHg}$ was used to ensure the minimal effect of cuff pressure on hemodynamics. Blood pressure was measured thereafter. CAVI was calculated by the following formula:

$$
\mathrm{CAVI}=\mathrm{a}\left\{(2 \rho / \Delta \mathrm{P}) \times \ln (\mathrm{Ps} / \mathrm{Pd}) \mathrm{PWV}^{2}\right\}+\mathrm{b}
$$

where Ps is systolic blood pressure, Pd is diastolic blood pressure, PWV is pulse wave velocity, $\Delta \mathrm{P}$ is Ps $\mathrm{Pd}, \rho$ is blood density, and $\mathrm{a}$ and $\mathrm{b}$ are constants.

Scale conversion was performed to compare CAVI with PWV (Hasegawas method). The VaSera was equipped with both measurement and calculation systems, and automatically calculated CAVI. The average coefficient of variation of CAVI is less than $5 \%$, which is sufficiently low for clinical usage and indicates that CAVI has good reproducibility ${ }^{12}$.

\section{Statistical Analysis}

Comparison between groups was performed using Student's $t$-test or the paired $t$-test. The relationship between changes in CAVI and each parameter was analyzed using simple regression analysis. In all comparisons, $p<0.05$ was considered significant.

\section{Results}

\section{Changes in Clinical Parameters After Pitavastatin Treatment}

The changes in clinical parameters after pitavastatin treatment are shown in Table 1. After pitavastatin administration for 12 months, significant decreases in TC and LDL-C and a significant increase in HDL-C were observed. No significant changes in body weight, BMI, blood pressure, HbA1c and TG were observed.

\section{Changes in 8-OHdG and MDA-LDL After 12} Months of Pitavastatin Treatment

The changes in urinary $8-\mathrm{OHdG}$ and serum MDA-LDL are shown in Fig. 1. After pitavastatin treatment for 12 months, significant decreases in 8-OHdG from 11.3 to $8.4 \mathrm{ng} / \mathrm{mg} \mathrm{Cr}(p<0.05)$ and MDA-LDL from 170 to $114 \mathrm{U} / \mathrm{L}(p<0.01)$ were observed.

\section{Change in CAVI After 12 Months of Pitavastatin Treatment}

The changes in CAVI are shown in Fig. 2. A significant decrease in CAVI from 9.54 to $8.91(p<0.05)$ was observed after 12 months of pitavastatin treatment.

\section{Correlation between Change in CAVI and Changes in Other Parameters}

Simple regression analyses were performed to 


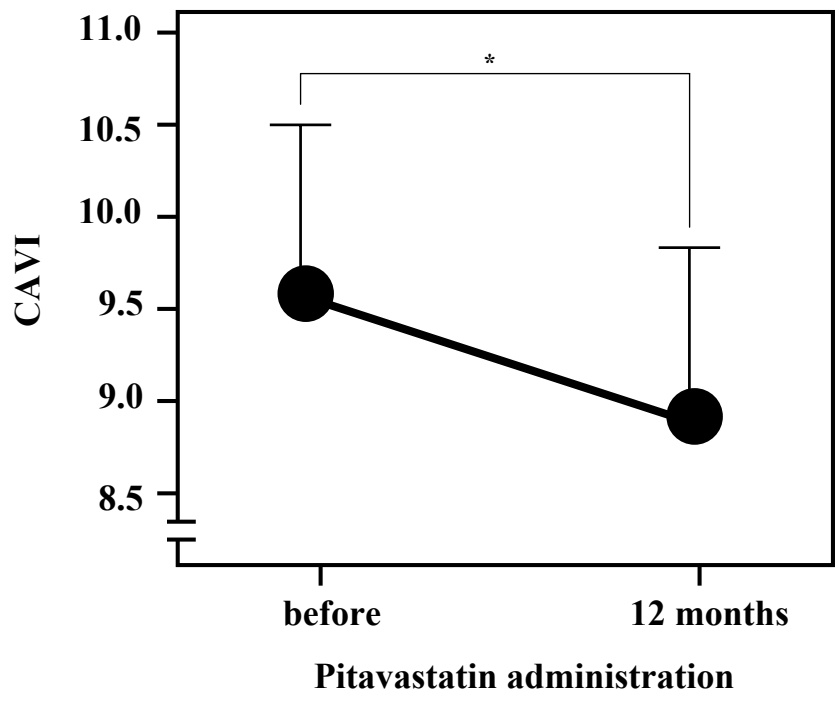

Fig. 2. Change in CAVI after pitavastatin treatment for 12 months.

Data are presented as the mean \pm S.D. ${ }^{*} p<0.05$ vs before treatment, paired $t$-test.

Table 2. Correlation between changes of CAVI and changes of parameters

\begin{tabular}{lcc}
\hline & $\begin{array}{c}\text { correlation } \\
\text { coefficient (R) }\end{array}$ & $p$ value \\
\hline$\Delta$ Hemoglobin A1c & 0.096 & 0.73 \\
$\Delta$ Total cholesterol & 0.089 & 0.72 \\
$\Delta$ Triglyceride & 0.184 & 0.45 \\
$\Delta$ HDL-cholesterol & 0.328 & 0.17 \\
$\Delta$ LDL-cholesterol & 0.249 & 0.39 \\
$\Delta$ Malondialdehyde-LDL & 0.549 & 0.02 \\
$\Delta$ 8-hydroxy-2'-deoxyguanosine & 0.104 & 0.67 \\
\hline
\end{tabular}

examine the correlation between changes in CAVI and changes in other parameters (Table 2). $\triangle$ CAVI correlated significantly with $\triangle \mathrm{MDA}-\mathrm{LDL}$. No significant correlation was observed between $\triangle$ CAVI and $\Delta \mathrm{LDL}-\mathrm{C}$.

Comparison of Background between CAVIImproved Group (Responders) and -Nonimproved Group (Non-Responders)

Subjects were divided into two groups: a group with improved CAVI $(\Delta$ CAVI $<0$; responders, $n=32)$ and a group with no improvement in CAVI $(\triangle \mathrm{CAVI}$ $\geqq 0$; non-responders, $n=13$ ). The baseline clinical profile of two groups and the changes in clinical parameters are shown in Table 3. Baseline levels of HbA1c, 8 -OHdG and CAVI in responders were significantly higher than in non-responders. TC and LDL-C fell significantly in both responders and non-responders; however, the changes in TC and LDL-C were not significantly different between groups (Table 3 ). Decreases in $8-\mathrm{OHdG}$ and MDA-LDL were greater in responders than in non-responders, and the change in MDA-LDL was significantly different between groups (Table 3).

\section{Discussion}

A novel arterial stiffness indicator, termed CAVI, has been developed recently as a noninvasive and easy technique for the diagnosis of atherosclerosis ${ }^{12-15)}$. In this study, we demonstrated that pitavastatin was effective in reducing 8-OHdG, MDA-LDL and CAVI in type 2 diabetes patients with high LDL-C, and that the change in CAVI correlated with the change in MDA-LDL. These results suggested that pitavastatin has the potential to reduce oxidative stress and may improve arterial stiffness by this potential.

In the diabetic state, increased production of reactive oxygen species and decreased antioxidant capacity have been observed, both resulting in increased oxidative stress ${ }^{19-21)}$. Enhanced oxidative stress may be closely related to the pathogenesis of atherosclerosis ${ }^{19,21,22)}$. In the present study, pitavastatin significantly decreased 8-OHdG, which is known to be a useful indicator of systemic oxidative stress ${ }^{23,24)}$. Pitavastatin has been reported to decrease vascular reactive oxygen (ROS) and nicotinamide adenine dinucleotide phosphate (NADPH) oxidase activity ${ }^{25,26)}$. These effects of pitavastatin may be reflected by a decrease in $8-O H d G$. Furthermore, increased oxidative stress and high levels of LDL may accelerate the production of oxidized LDL. The present results suggest that pitavastatin may decrease MDA-LDL, which is known to be a form of oxidized LDL ${ }^{27,28)}$, by two major mechanisms: a systemic oxidative stress-ameliorating effect and a LDLC-lowering effect.

There are at least two possible explanations for the mechanisms of CAVI reduction. The first is regression of a vascular organic lesion. Recent reports indicate that CAVI is capable of detecting the presence of a vascular organic lesion ${ }^{12-15)}$, which might regress as a result of reduced oxidative stress. For example, Tani et al. ${ }^{29)}$ demonstrated the regression of coronary atherosclerosis by reducing MDA-LDL by $12.7 \%$. Ono et al. ${ }^{30)}$ reported that carotid intima-media thickness was decreased by reducing 8 -OHdG from 10.98 to 7.61 $\mathrm{ng} / \mathrm{mg}$ Cr. In the present study, the magnitudes of decrease in MDA-LDL (33\%) and 8-OHdG (11.3 to $8.4 \mathrm{ng} / \mathrm{mg} \mathrm{Cr}$ ) were comparable or better than in pre- 
Table 3. Comparison of background between responders and non-responders

\begin{tabular}{|c|c|c|c|c|}
\hline & \multicolumn{2}{|c|}{ Responders } & \multicolumn{2}{|c|}{ Non-responders } \\
\hline & before & after & before & after \\
\hline n (male/female) & $32(14 / 18)$ & & $13(5 / 8)$ & \\
\hline Age & $65.6 \pm 6.3$ & & $65.3 \pm 6.3$ & \\
\hline BMI $\left(\mathrm{kg} / \mathrm{m}^{2}\right)$ & $22.7 \pm 1.8$ & $22.4 \pm 1.5$ & $22.2 \pm 1.4$ & $22.1 \pm 1.2$ \\
\hline \multicolumn{5}{|l|}{ Blood pressure $(\mathrm{mmHg})$} \\
\hline systolic & $124 \pm 11$ & $125 \pm 12$ & $126 \pm 12$ & $126 \pm 13$ \\
\hline diastolic & $77 \pm 8$ & $75 \pm 7$ & $78 \pm 7$ & $76 \pm 8$ \\
\hline Hemoglobin A1c (\%) & $7.3 \pm 1.4$ & $6.8 \pm 1.1$ & $6.4 \pm 1.1^{*}$ & $6.3 \pm 0.9$ \\
\hline Total cholesterol (mg/dL) & $245 \pm 26$ & $202 \pm 21^{* *}$ & $247 \pm 21$ & $205 \pm 24^{* *}$ \\
\hline Triglyceride $(\mathrm{mg} / \mathrm{dL})$ & $151 \pm 47$ & $140 \pm 51$ & $140 \pm 53$ & $139 \pm 42$ \\
\hline HDL-cholesterol (mg/dL) & $52.7 \pm 11.2$ & $56.6 \pm 9.3$ & $50.4 \pm 12.1$ & $53.0 \pm 10.6$ \\
\hline LDL-cholesterol (mg/dL) & $167 \pm 23$ & $127 \pm 25^{* *}$ & $164 \pm 25$ & $128 \pm 26^{* *}$ \\
\hline Malondialdehyde-LDL (U/L) & $178 \pm 52$ & $106 \pm 37^{* *}$ & $150 \pm 27$ & $132 \pm 28^{* *}$ \\
\hline 8-hydroxy-2'-deoxyguanosine (ng/mg Cr) & $12.6 \pm 6.1$ & $8.8 \pm 3.2^{* *}$ & $8.9 \pm 5.8^{*}$ & $7.0 \pm 2.8^{* *}$ \\
\hline Cardio-ankle vascular index & $9.79 \pm 1.21$ & $8.88 \pm 1.1$ & $8.92 \pm 1.1^{*}$ & $8.98 \pm 0.4$ \\
\hline \multicolumn{5}{|l|}{ Anti-diabetic agents [no. of subjects (\%)] } \\
\hline Sulfonylureas & $24(75 \%)$ & & $8(62 \%)$ & \\
\hline Thiazolidinedione & $3(9 \%)$ & & $1(8 \%)$ & \\
\hline Alpha-glucosidase inhibitor & $6(19 \%)$ & & $4(31 \%)$ & \\
\hline Biguanide & $10(31 \%)$ & & $6(46 \%)$ & \\
\hline Smoking [no. of subjects (\%)] & $2(6 \%)$ & & $1(8 \%)$ & \\
\hline
\end{tabular}

Data: mean \pm S.D.

${ }^{*} p<0.05$ vs responders, Student's $t$-test. ${ }^{* *} p<0.05$ vs before. Student's $t$-test.

vious studies. Thus, the reduction of CAVI observed in this study may well reflect the improvement of a vascular organic lesion as a result of significant decreases in MDL-LDL and 8-OHdG. Another possible mechanism is improved endothelial function. Among mega trials with statins, the ASTEROID trial (A Study To evaluate the Effect of Rosuvastatin On Intravascular ultrasound- Derived coronary atheroma burden) demonstrated that a decrease in LDL-C to $60.8 \mathrm{mg} / \mathrm{dL}$ by very high-intensity statin therapy resulted in a significant reduction of plaque volume in patients with coronary heart disease confirmed by coronary angiography ${ }^{31)}$. In the present study, the reduction of LDL-C did not reach the level achieved in the ASTEROID trial. Furthermore, our study duration was 12 months compared with 2 years in ASTEROID. Thus, from the viewpoint of the LDL-C-reducing effect, pitavastatin treatment for 12 months is unlikely to have regressed atherosclerotic lesions in our patients. It has been reported that oxidized LDL and 8-OHdG contribute to atherogenetic processes by inducing vascular endothelial dysfunction ${ }^{32,33)}$. Therefore, the improvement in CAVI observed in our study may be consistent mainly with the improvement of endothe- lial dysfunction but not the regression of a organic lesion. Endothelial function is known to be affected by many factors, especially by dietary conditions ${ }^{34,35)}$; therefore, further studies to clarify the effects of these factors are required in the future.

In this study, the patients were divided into two groups: one group showed improved CAVI (responders), and the other group showed no improvement in CAVI (non-responders). The responders showed a significantly greater decrease in MDA-LDL than nonresponders, although the decreases in LDL-C were almost identical in the two groups. To clarify the characteristics of responders and non-responders, we compared the baseline data of the two groups. Serum HbA1c, urinary 8-OHdG and the CAVI were significantly higher in responders than in non-responders. These findings suggest that the potential of pitavastatin to ameliorate oxidative stress may be enhanced in a state of deteriorated metabolic disorder, and this potential may be independent of the lipid-lowering action. CAVI is known to correlate positively with age and has different normal values in males and females ${ }^{12}$. In the present study, age and the male/female ratio were not significant difference in responder and non- 
responder groups; therefore, these factors were considered to have had no effect on the results.

Diabetes mellitus is a very important risk factor for atherosclerosis. Atherosclerotic diseases are a common cause of sudden death, partially due to the lack of a simple and reliable noninvasive diagnostic method of atherosclerosis that can be used routinely in clinical settings. In the present study, CAVI was improved by pitavastatin treatment accompanied by a reduction of oxidative stress. From these findings, we conclude that pitavastatin may be effective to improve arterial stiffness in patients with enhanced oxidative stress, and that CAVI may be useful as a routine test for the diagnosis and therapeutic monitoring of atherosclerosis. A large-scale prospective study to evaluate CAVI and the effectiveness of intensive statin therapy is warranted.

\section{References}

1) Lyons TJ: Oxidized LDL: a role in the pathogenesis of atherosclerosis in diabetes? Diabetic Med, 1991; 8: 411419

2) Kannnel WB, McGee DL: Diabetes and cardiovascular disease, the Framingham study. JAMA, 1979; 241: 2035 2038

3) Shepherd J, Cobbe SM, Ford I, Isles CG, Lorimer AR, Macfarlane PW, Mckillop JH, Packard CJ: Prevention of coronary heart disease with pravastatin in men with hypercholesterolemia. N Engl J Med, 1995; 333: 13011307

4) Pitt B, Waters D, Brown WV, van Boven AJ, Schwartz L Title LN, Eisenberg D, Shurzinske L, MacCormick LS: Aggressive lipid-lowering therapy compared with angioplasty in stable coronary artery disease. Atorvastatin versus Revascularization Trectment Investigators. N Engl J Med, 1999; 341: 70-76

5) Byington RP, Furberg CD, Crouse JR 3rd, Espeland MA, Bond MG: Pravastatin, Lipids, and atherosclerosis in the carotid arteries (PLAC-II). Am J Cardiol, 1995; 76: 54C$59 \mathrm{C}$

6) Bramwell JC, Hill AV: Velocity of the Pulse wave in Man Proc Roy Soc, 1922; B: 298-306

7) Hayashi G, Sato M, Niimi H, Handa H, Moritake K, Okumura A: Analysis of vascular wall constitutive law with finite deformation theory. Medical Electronics and Biological Engineering, 1975; 13: 293-297

8) Hasegawa M: Fundamental research on human aortic pulse wave velocity. Jikei Medical Journal, 1970; 85: 742760

9) Asmar R: Pulse wave velocity principles and measurement 25-55, in Arterial stiffness and pulse wave velocity edited by Asmar R, O’Rourke MF, Safar M, Elsevier Amsterdam, 1999

10) Kawasaki A, Takeuchi K, Hasegawa M, Yagi S, Nakayama S, Takayama Y: Noninvasive measurement of common carotid artery effect with echo phase tracking system. Journal of Japanese College of Angiology, 1982; 22: 241-
248

11) Buntin CM, Silver FH: Noninvasive assessment of mechanical properties of peripheral arteries. Ann Biomed Eng, 1990; 18: 549-566

12) Shirai K, Utino J, Otsuka K, Takata M: A novel blood pressure-independent arterial wall stiffness parameter; cardio-ankle vascular index (CAVI). J Atheroscler Thromb, 2006; 13: 101-107

13) Yambe T, Meng X, Hou X, Wang Q, Kekine K, Shiraishi Y, Watanabe M, Tamaguchi T, Shibata M, Kuwayama T, Maruyama M, Konno S, Nitta S: Cardio-ankle vascular index (CAVI) for the monitoring of the atherosclerosis after heart transplantation. Biomed Pharmacother, 2005; 59(Suppl 1): S177-S179

14) Kubozono T, Miyata M, Ueyama K, Nagaki A, Otsuji $Y$, Kusano K, Kubozono O, Tei C: Clinical significance and reproducibility of new arterial distensibility index. Circ J, 2007; 71: 89-94

15) Nakamura K, Tomaru T, Yamamura S, Miyashita Y, Shirai $\mathrm{K}$, Noike H: Cardio-ankle vascular index is a candidate predictor of coronary atherosclerosis. Circ J, 2008; 72: 598-604

16) Shirai $K$, Nema T, Hiroh $Y$, Itoh $Y$, Miyashita $Y$, Watanabe $\mathrm{H}$ : Clinical efficacy of the direct assay method using polymers for serum high density lipoprotein cholesterols. J Clin Lab Anal, 1992; 11: 82-86 (a)

17) Kondo A, Muranaka Y, Ohta I, Notsu K, Manabe M, Kotani K, Saito K, Maekawa M, Kanno T: Relationship between triglyceride concentrations and LDL size evaluated by malondialdehyde-modified LDL. Clin Chem, 2001; 47: 893-900

18) Toyokuni $S$, Tanaka T, Hattori $Y$, Nishiyama $Y$, Yoshida A, Uchida K, Hiai H, Ochi H, Osawa T: Quantitative immunohistochemical determination of 8-hydroxy-2'deoxyguanosine by a monoclonal antibody N45.1: its application to ferric nitrilotriacetate-induced renal carcinogenesis model. Lab Invest, 1997; 76: 365-374

19) Baynes JW: Role of oxidative stress in development of complication in diabetes. Diabetes, 1991; 40: 405-412

20) Lyons TJ: Oxidized LDL: a role in the pathogenesis of atherosclerosis in diabetes? Diabetic Med, 1991; 8: 411419

21) Giugliano D, Ceriello A, Paolisso G: Oxidative stress and diabetic vascular complications. Diabetes Care, 1996; 19: 257-267

22) Baynes JW, Thorpe SR: The role of oxidative stress in diabetic complications. Curr Opin Endocrinol, 1997; 3: 277-284

23) Shigenaga MK, Gimeno CJ, Ames BN: Urinary 8-hydroxy2'-deoxyguanosine as a biological marker of in vivo oxidative DNA damage. Proc Natl Acad Sci USA, 1989; 86: 9697-9701

24) Nishikawa T, Sasahara T, Kiritoshi S, Sonoda K, Senokuchi T, Matsuo T, Kukidome D, Wake N, Matsumura T, Miyamura N, Sakakida M, Kishikawa H, Araki E: Evaluation of urinary 8-hydroxydeoxy-guanosine as a novel biomarker of macrovascular complications in type 2 diabetes. Diabetes Care, 2003; 26: 1507-1512

25) Chinen I, Shimabukuro M, Yamakawa K, Higa N, Matsuzaki T, Noguchi K, Ueda S, Sakanashi M, Takasu N: 
Vascular lipotoxicity: endothelial dysfunction via fattyacid-induced reactive oxygen species overproduction in obese Zucker diabetic fatty rats. Endocrinology, 2007; 148: $160-165$

26) Umeji K, Umemoto $S$, Itoh $S$, Tanaka $M$, Kawahara $S$, Fukai T, Matsuzaki M: Comparative effects of pitavastatin and probucol on oxidative stress, $\mathrm{Cu} / \mathrm{Zn}$ superoxide dismutase, PPAR-gamma, and aortic stiffness in hypercholesterolemia. Am J Physiol Heart Circ Physiol, 2006; 291: $\mathrm{H} 2522-2532$

27) Kondo A, Li J, Manabe M, Saito K, Kanno T, Maekawa M: Relationship between high-density lipoprotein-cholesterol and malondialdehyde-modified low-density lipoprotein concentrations. J Atheroscler Thromb, 2003; 10: 72-78

28) Kondo A, Manabe M, Saito K, Maekawa M, Kanno T: Insulin treatment prevents LDL from accelerated oxidation in patients with diabetes. J Atheroscler Thromb, 2002; 9: $280-287$

29) Tani S, Watanabe I, Anazawa T, Kawamata H, Tachibana E, Furukawa K, Sato Y, Nagao K, Kanmatsuse K, Kushiro T: Surusadai Atherosclerosis Regression Investigators Effect of pravastatin on malondialdehyde- modified low-density lipoprotein levels and coronary plaque regression as determined by three-dimensional intravascular ultrasound. Am J Cardiol, 2005; 96: 1089-1094

30) Ono H, Minatoguchi S, Watanabe K, Yamada Y, Mizukusa T, Kawasaki H, Takahashi H, Uno T, Tsukamoto T, Hiei K, Fujiwara H: Candesartan decreases carotid intima- media thickness by enhancing nitric oxide and decreasing oxidative stress in patients with hypertension. Hypertens Res, 2008; 31: 271-279

31) Nissen SE, Nicholls SJ, Sipahi I, Libby P, Raichlen JS, Ballantyne CM, Davignon J, Erbel R, Fruchart JC, Tardif JC, Schoenhagen P, Crowe T, Cain V, Wolski K, Goormastic M, Tuzcu EM: ASTEROID Investigators Effect of very high-intensity statin therapy on regression of coronary atherosclerosis: the ASTEROID trial. JAMA, 2006; 295: 1556-1565

32) Fan J, Watanabe T: Inflammatory reactions in the pathogenesis of atherosclerosis. J Atheroscler Thromb, 2003; 10: 63-71

33) Lei YC, Hwang JS, Chen CC, Lee CT, Cheng TJ: Enhanced oxidative stress and endothelial dysfunction in streptozotocin-diabetic rats exposed to fine particles. Environ Res, 2005; 99: 335-343

34) Shimabukuro M, Chinen I, Higa N, Takasu N, Yamakawa K, Ueda S: Effects of dietary composition on postprandial endothelial function and adiponectin concentrations in healthy humans: a crossover controlled study. Am J Clin Nutr, 2007; 86: 923-928

35) Wascher TC, Schmoelzer I, Wiegratz A, Stuehlinger M, Mueller-Wieland D, Kotzka J, Enderle M: Reduction of postchallenge hyperglycaemia prevents acute endothelial dysfunction in subjects with impaired glucose tolerance. Eur J Clin Invest, 2005; 35: 551-557 